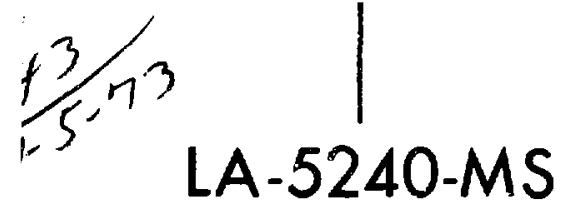

INFORMAL REPORT

MCP Code Fluorescence-Routine Revision

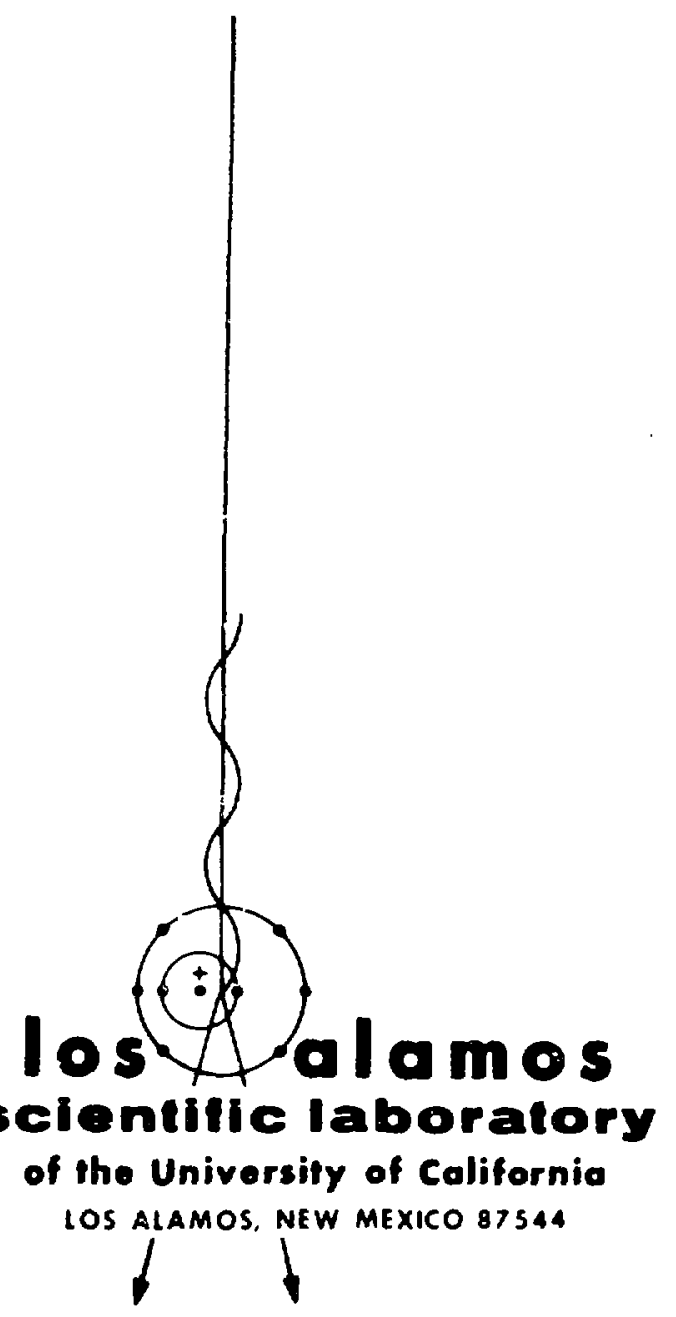


This report was prepared as an account of work sponsored by the United States Government. Neither the United States nor the United States Atomic Energy Commission, nor any of their employees, nor any of their contrac. tors. subcontractors, or their employees, makes any wartanty. express or im. plied. or assumes any legai liability or pesponsibility for the accuracy. com. pleteness or usefulness of any information. apparatus, product or process dis. closed, or represents that its use would not infringe privately owned rights.

In the interest of prompt distribution. this LAMS re. port was not edited by the Technical Information staff.

Frunied in the United States af America. Avalabile from National Technucal Information Service

U. S. Depariment of Cominetce

5265 Porl Floval Road

Soringlield. Virginia 22151

Price: Printed Copy Sys: Nicrentiche 50.95

$$
+\not 1.00
$$


LA.5240.MS

Informa: Peport

UC. $32 \& 34$

ISSUED: May 1973

\section{MCP Code Fluorescence-Routine Revision}

by

C. J. Evarett

E. D. Cashwell

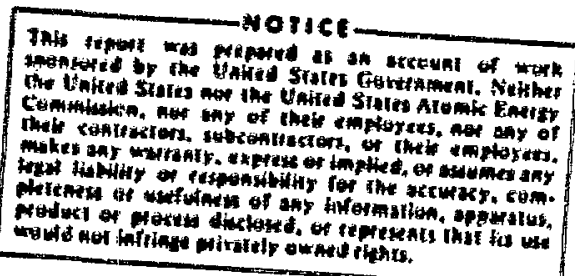


HCP CODE FUUORESCEACE-ROUTIHE REVISION

by

C. J. Evereze and E. D. Gastruel1

\begin{abstract}
AsSTRACT
A nes wathod is deser lbed for zreating fluorescence, whlch replaces the original subrourine of the Honce Carlo photon code (MCP). and ellmintes tes wose undesirable features. The rajor changes include (a) eliatnacion of the very incecurate $1 / 8^{3}$ lav for phozo- lectile cross section. (b) updating of relative infensities of K-fluorescence 11res. (c) eltmination of tuorescence frow shells othar than $x$ and $b$, and (d) provision for secondary $L-f$ luorescence. Except for the lacter, the provlous gcde 18 unchanged, as rell as the atorage forant. Hovever, the change in zethod is retilected in the ney contente, whtch have been cospletely revised, and are included here in rebulated fora.
\end{abstract}

\section{IMTrodetetict}

The reviaton of the origlinal fluoreseence ereatment in the WCP code, 1 tesertbed below, is destgred

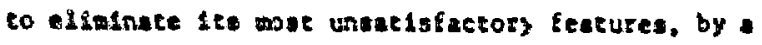
sechod inwoluing only a slighe addtcion to the prevloue routine. but an lmose cocal replacesent of the convants in "z-section F" of the supporting Itbrary zape. The eppended tebles, now cosplete for all elementa $12<2<94$, are Iderelcal in forwhe with the extselis daze of that section. except for sowe reductions in the length $f$ tor $2>70$. These reductione erat due to the eliminacion of cluorescence fros shells ocher then $x$ and $k$. The probabllicy of ejection from ocher shells, as vell as the corresponding ylelds and fluozesent energies, are all comarastvely sall, and ore greatest for high 2 , where the p.e. cross section is enorows at che Ifurescent energits. He therefore assune lotal ubsorption for auch fluoreseence.

Civen a photoliectric cvent. the puzpose of the present fluareacence subrourine is cherefore co deterolne frow uhtch of the two shells, $x$ or 1. an election to efeced (if of thes), and the fluoreseene photon energy enteted (If any).
In F1g. 1, we give for essy reference in overvies of the data to be discusged, and upon whleh Table I is based.

11. EDGE ENERGIES

The single $k$-edge energy $E_{K}$ is caken frod Iable II of Ref. 2, betng ldentleal with that of Ref. 3, which was used before. The L-edge enezhy (for $z>11$ ) 1 a regarded so the simple avezage

$$
E_{L}-\left(E_{21}+E_{12}+E_{13}\right) / 3
$$

the Indluiduel $\varepsilon_{t, 1}$ being those in Table Il of Ref. 2 . The energles $E_{K}, F_{L}$. compared $w 1$ th the Ineldent photon energy $x$, serve to deteretne the possiblitiy of $x$ or t. ejectlons. The $1,-$ shell in treated as ande utth respect to primary 1,-fluorescence.

\section{IIT. FLUORESGENT ENERCIES}

Upon ejection of an eluseron fros a shell of encrgy $E_{S}$ by an incident photon $\left(E>E_{E}\right.$ ) ehc vaeancy czesced is fliled by an electron transietion fros an "outer" shell of energy $E_{T}<E_{5}$. The photon, of energy $F=E_{S}-E_{T}$, ereaced in the cranstelon, wy or wy not cscape the atom. In the former caso, it is referzed to as (primary) S-fiureacence. 


\begin{tabular}{|c|c|c|c|c|c|}
\hline 2 & e & $\phi$ & $x$ & $F$ & $\underline{f}$ \\
\hline \multirow[t]{2}{*}{$12-19$} & $E_{R}$ & $\phi_{0}=D_{R}$ & $Y_{0}=0$ & 0 & 2 \\
\hline & $\varepsilon_{k}$ & $\phi_{K}=1-\rho_{K}$ & $Y_{K}$ & $F_{K}$ & \\
\hline \multirow[t]{4}{*}{$20-30$} & $E_{K}$ & $\phi_{0}=P_{x}$ & $\gamma_{0}=0$ & 0 & 4 \\
\hline & $E_{K}$ & & ${ }^{Y_{K} P_{1}}$ & $\mathrm{FRa}_{1}$ & \\
\hline & $E_{K}$ & & $\mathbf{x}_{x x_{2}}$ & $\mathrm{FKa}_{2}$ & \\
\hline & $E_{k}$ & $o_{K}=1-\rho_{K}$ & ${ }^{x^{p_{3}}}$ & FKB ${ }_{1}^{-}$ & \\
\hline \multirow[t]{5}{*}{$31-36$} & $\overline{\mathbf{E}}_{\mathbf{L}}$ & 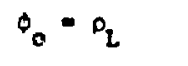 & $Y_{0}=0$ & 0 & 5 \\
\hline & $\overline{\mathrm{E}}_{2}$ & $\theta_{L}=1-p_{L}$ & $\mathbf{Y}_{\mathbf{L}}$ & $F_{2}$ & \\
\hline & $\mathbf{E}_{K}$ & & $\mathbf{Y}_{R} P_{I}$ & $\mathrm{FKa}_{1}$ & \\
\hline & $\mathbf{z}_{\mathrm{K}}$ & & ${ }^{Y_{k} \mathbf{p}_{2}}$ & $\mathrm{FKE}_{2}$ & \\
\hline & $E_{K}$ & $\theta_{K}=\frac{t_{0}}{\rho_{K}}-1$ & ${ }^{\mathbf{X} \mathbf{P}_{3}}$ & $\mathrm{FKB}_{1}^{\prime}$ & \\
\hline \multirow[t]{6}{*}{$37-94$} & $\overline{\mathbf{E}}$ & $\theta_{0}=p_{2}$ & $Y_{0}=0$ & 0 & 6 \\
\hline & $\overline{\mathrm{E}_{2}}$ & $\phi_{L}=1-p_{2}$ & $\mathbf{Y}_{\mathbf{L}}$ & $F_{L}$ & \\
\hline & $E_{R}$ & & $Y_{k} P_{I}$ & $\mathrm{FXa}_{1}$ & \\
\hline & $\mathbf{E}_{\mathbf{K}}$ & & ${ }^{Y_{k} p_{2}}$ & $\mathrm{EKC}_{2}$ & \\
\hline & $\varepsilon_{K}$ & & ${ }^{Y_{K} \mathbf{P}_{3}}$ & $\mathrm{FKB}_{1}^{-}$ & \\
\hline & $E_{x}$ & $\phi_{\mathrm{K}}=\frac{1}{\hat{\theta}_{\mathrm{K}}}-1$ & $\mathbf{Y}_{K^{P_{4}}}$ & $\mathrm{FKB}_{2}^{-}$ & \\
\hline
\end{tabular}

Fig. I. Overvier of the data.

L-fluoreacence is allowed only for $2>31$, and the single fluorescent energy $F_{L}$ is tsken as the i.123 in Table $v$ of pef. 2. Th1s is an average of all energy cops from etges $\mathrm{Mj}$. Nk, ... to $a 11$ LI edges, velghted by their ralative 10tandtes, is civen in Fables IV and VI of Ref. 2 .

The olngle value $F_{K}$ eiven for $12<z<19$ 1s the weighted average of the $\mathrm{Ka}_{1}, \mathrm{Ka}_{2}$ Lines, siven as $\bar{R} a$ in Table $v$ of heE. 2.

For $20<2<94$, the individual fluorescent energles $\mathrm{FKa}_{1}>\mathrm{FKa}_{2}$ are saken from Table III of Ref. 2. These are pure lines resulting fron the zeanatzione $2.3 \rightarrow x, 2.2+x$, respeczively. For $20<2<94$, the fluorescent energy $\operatorname{FXB}_{1}^{\prime}\left(H 2 \rightarrow x_{1}\right.$ $H 3+K, M 4+K$, composite) is al*o al lowed end 10 taken as the velghted sverage of the three entrgy differences, conputed from Tables III, VI of Ref. 2 .

For $37<z<94$, the cebulated energy $\mathrm{FKB}_{2}^{\circ}$ (W2 $\rightarrow \mathrm{K}, \mathrm{N} 3 \rightarrow \mathrm{K})$ 1s the corrasgonding uetghed

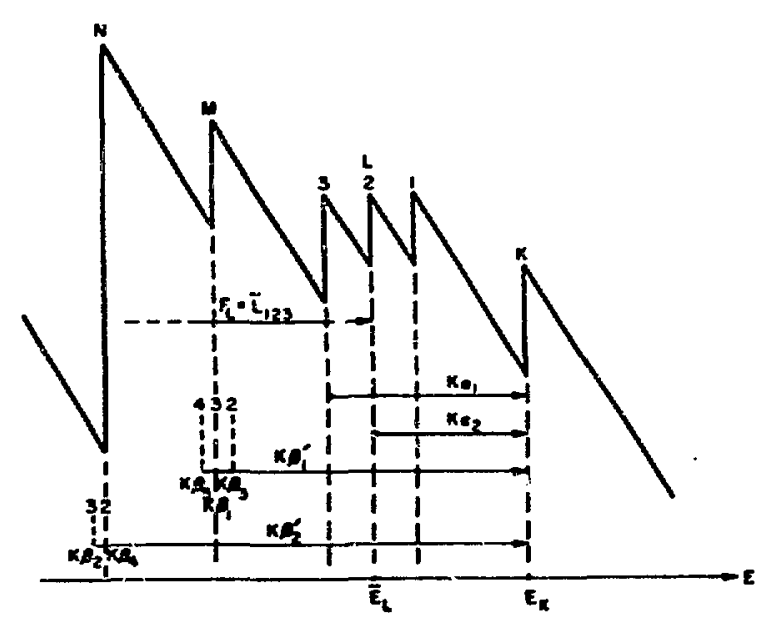

P1g. 2. Fluorescent "L1nes".

average of these two lines, obtalned in the same way.

Noce the order of tabulation in F1g. 1 and the reiations

$$
\begin{aligned}
& 12<z<19 . \bar{K}(r) \text { Av. }\left(K a_{1}, K a_{2}\right) \\
& 20<2<30 . \mathrm{ka} a_{1}, \mathrm{ka} a_{2}, \mathrm{kB}{ }_{1}^{-} \\
& 31 \sigma_{2}<36 . \overline{\mathrm{t}}_{123} \mathrm{Av}, \mathrm{Ka_{1 }}, \mathrm{Ka}, \mathrm{Ka} \\
& 37<2<94 . i_{123} A v ., k a_{1}, k a_{2}, K B_{1}^{j}, \pi B_{2}^{-} \\
& F_{L}<\mathrm{FXO}_{2}<\mathrm{FKa}_{1}<\mathrm{FKB}_{1}<\mathrm{FKB}_{2}^{\prime}
\end{aligned}
$$

The fluorescent "lines" provided for are Indiceted very sehemaslcally in F1B. 2 .

\section{THE YIELDS $Y$}

The yleld $Y_{S}$ for a shell $S$ is the total probebll1zy of fluorescent enisalon acsomparying electron transition frow all outer shells to a vacancy in thell $S$, hovever created (CE. Pare VII).

The date for $y_{2}$ in Ref. $4(2>31)$ are very spotty; and the valuet nov used, froe Tabie VIII of Ref. 1, seen noe coo bed a compromlse between those of Ref. 4 and those of Ref. 5, which vere used previousky.

The sotal yield $Y_{x}$ is thet used before, furnished by larael and storm as an updesed veralon of Table vili of Ref. 3, For th1s, we have no publiahed 
reference. (№n ylelds are included in Ref. 2.) We note that the update is in general accord with Table II of Ref. 4 and for $Z>60$ is 1dentical with Ref. 3, but 18 higher for $2<60$.

As noted in Part III, K-fluorescence was asswoed to consist of the three 11 res $\mathrm{Ka}_{1}, \mathrm{Ka}_{2}, \mathrm{KH}_{1}^{-}$for $20<z<36$ and for $z>36$ of the additional line $\mathrm{RB}_{2}$. These assumptions were made before, but the relative Intenalties of the lines were based on Ref. 6. We now use the Intensities given in Table VI of Ref. 2, which were based on the calculations of Scofield and are considered to be nearer the truth. Fron thees data, the probabilities $P_{1}, P_{2}$, $P_{3}(20<2<36)$ and $P_{1}, \ldots, P_{4}(z>36)$ of the components of the yield $Y_{K}$ were obtained, and thus the Individital yields as indicated in Fig. 1.

\section{RELATIVE PROBABILITIES OF $K, l$ EJECTION}

A. For $12<2<30$

Only k-fluorescence is consldered and can occur only for $E>E_{K}$. For such an incidert $E$, te 1. assueed (cf. Ref. 2, p. 569) that the probability of a k-ejection has the constant vaiue

$$
\phi_{X}=\left(\sigma_{K}-\sigma_{x}^{\prime}\right) / \sigma_{k}=1-\rho_{K}
$$

where $o_{X}=\sigma_{K}^{-} / \sigma_{K}$ is the ratio of the p.e. cross section at botton and top of the K-edge, as Ind1cated In FIg. 3. Here and elsevhere, $\sigma^{*}$ and $\sigma$ are saken from Table $I$ of Ref. 2 . Note that $p_{K} 1$ s the entry $a_{K}$ (photo)/o(photo) given in table virz. Referring to F1s. 1, it 1 clear that $\phi_{K} Y_{K}$ is the probab1liey of fluorescence $F_{K}$ for $12<z<19$, wh1le, for example, ${ }_{X} Y_{X} P_{1}$ is the probab121ty of FKa 1 tluorescence if $20<2<30$, aecusing a.e. went at $E>E_{k}$.

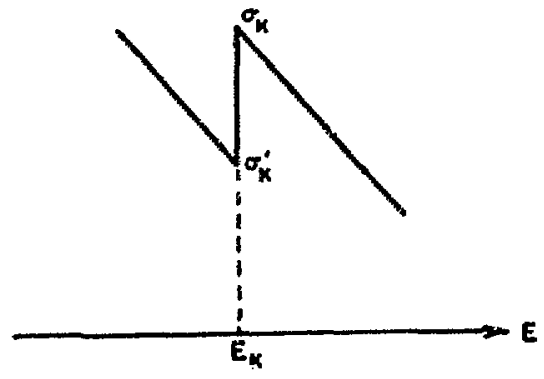

F18. 3. K-edge atructure.

\section{d. For $31 \leq 2<94$}

Both $L$ ind $K$ ejections are considered; and In order to follow the scheme of Ref. 5 used before, with no change in code, we require three numbers

$$
\phi_{R}, \phi_{L}, \phi_{0}
$$

which will deterralne the relat1ve probab1lities of $K, L$, and outer shall ejection for p.e. events at $E>E_{K}$, and such that $\phi_{L}, \phi_{0}$ also define the chances of $\mathrm{L}$ or outer shell ejection for $\overline{\mathrm{E}}_{2}<\mathrm{E}<\mathrm{E}_{\mathrm{R}}$.

Our basic assumption here 18 that the relative contribution of any edge so the total p.e. $\sigma$ at that edge $18\left(\sigma-\sigma^{\prime}\right) / \sigma=1-\sigma^{\prime} / \sigma$, and that th1s contr1bution rewains constant up to the next edge of higher energy (1f any). If we define $p_{1} \approx \sigma_{1}^{i} / \sigma_{1}, 1=1,2,3$ (see Fig. 4), it is then easy to show that

$$
\rho_{\mathrm{L}} \equiv \rho_{1} \rho_{2} \rho_{3} \quad \text { and } \quad 1-p_{\mathrm{L}}
$$

are the probabilities of outer and $L$ shell ejectiona at the $L_{1}$ edge. (The lacter appears in colum 2 of Table VIII of Ref. 2, with some winor c1screpancies.) S1a12erly, with $\rho_{K} \equiv \sigma_{\mathrm{g}}^{-} / \sigma_{K}$, one sees that $p_{1} \sigma_{K}^{\prime} / \sigma_{K}=\rho_{L} \rho_{K},\left(1-\rho_{L}\right) \sigma_{K}^{-} / \sigma_{K}=\left(1-\rho_{L}\right) o_{K}$, $\left(\sigma_{K}-\sigma_{K}\right) / \sigma_{K}+1-o_{K}$ are the probabilities of outex, L. and $X$ efections at the $\mathrm{K}$-edge. The proportional numbers

$$
\phi_{0}=p_{Z}, \theta_{L}-1-p_{L}, \phi_{K}=\frac{1}{p_{K}}-1
$$

also define these probabilities, when normed by their sum $1 / \%_{K}$, ano moreover the flrst two are preclsely

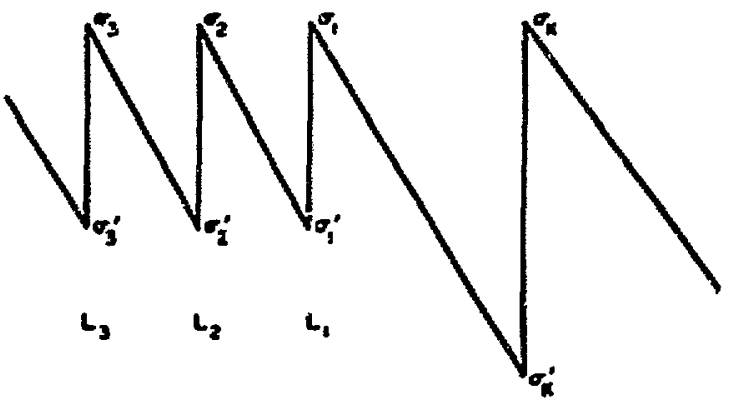

Fsg. 4. L and $K$ edge structure. 
the probabilities in (1). The numbers (2) therefcre satisfy our requirements and appear in the $\phi$ column of Fig. 1 for $z \geqslant 31$.

The bastc assumptions adopted above constitute a radical departure from the method of Ref. 5 , used before. The latter Involved essentially the same procedure, but supposed the minimal $\sigma_{t}^{\prime}$ value at an edge $t$ given by $\sigma_{t}^{-}=\sigma_{t+1} E_{t+1}^{3} / E_{t}^{3}$, where $t+1$ is the next edge of lower energy. The underlylng $1 / \mathrm{E}^{3}$ law Is very inaccurate, and it seens that the present wethod 1a auperior, insofar as the values of $\sigma^{-}, \sigma$ are correct in Table I of Ref. 2.

VI. THE FIRIAL TABULATED $\Phi$ AND $Y$

In order to facilitate computation, the values i1sted as $\phi$ and $Y$ in the $f$ inal appended tables were derived from the entries in Fig. 1 as indicaced in Fig. 5. The tabulation for $37<z<94$ is the exact analogue of that for $31<z<36$, and is onitted.

Two exaxples, for $31 \leqslant z<36$, should make the method clear: (1) for a p.e. event at $\varepsilon \geqslant E_{K}$, a random number between $Y_{4} /\left(\phi_{0}+\phi_{L}+\phi_{K}\right)$ and $y_{3} /$ $\left(\phi_{0}+\phi_{L}+\phi_{K}\right)$ implles a $F K a_{2}$ fluorescence; (2) for $E_{L}<\mathrm{E}<E_{K}$, a random number between $Y_{2} /\left(\phi_{0}+\phi_{L}\right)$ and $Y_{1} /\left(\phi_{0}+\phi_{L}\right)(=0)$ implies an $F_{L}$ fluorescence.

\section{SECONDARY L-FLLORESCENCE}

The fluorescence thus far discussed is primary, In the sense that 1 arises from the transition of an electron from an outer shell to a shell in which a vacancy has been created by ejection of an electron from that shell by the Initial incident photon. Thus, we have allowed for $\mathrm{K}-\mathrm{fl}$ luorescenct following a $\mathrm{K}$ ejection, and for L-fluorescence following an Iefection. Only such primary fluorescence was considered in the original code.

In this final section, we attempt to show roughly why secondary L-fluorescence may be of the same order of importance as primary L-fluorscence, and then describe how the present code provides for it, In a necessarily approximate fashion.

We stipulate a p.e. eveat on an element $z>31$, at an Incident energy $E>E_{K}$. Then the probability of an L-ejection is $\phi_{L} / \Sigma$, where $\Sigma=\phi_{0}+\phi_{L}+\phi_{K}$, and, as already stated, the overall probabllity of primary L-fluorescence ls given by

$$
P^{-}=\frac{\phi_{L}}{\Sigma} \cdot Y_{L}
$$

On the other hand, there $1 s$ a probability $\phi_{\mathrm{h}} / \Sigma$ of a $\mathrm{K}$-ejection. In this event, the $\mathrm{K}$-vacancy may be filled by an $\mathrm{L} 3+\mathrm{K}$ or $\mathrm{L} 2 \rightarrow \mathrm{K}$ transition, say with probabllities $P_{L 3 K}, P_{L 2 K}$, thus creating a vacancy in

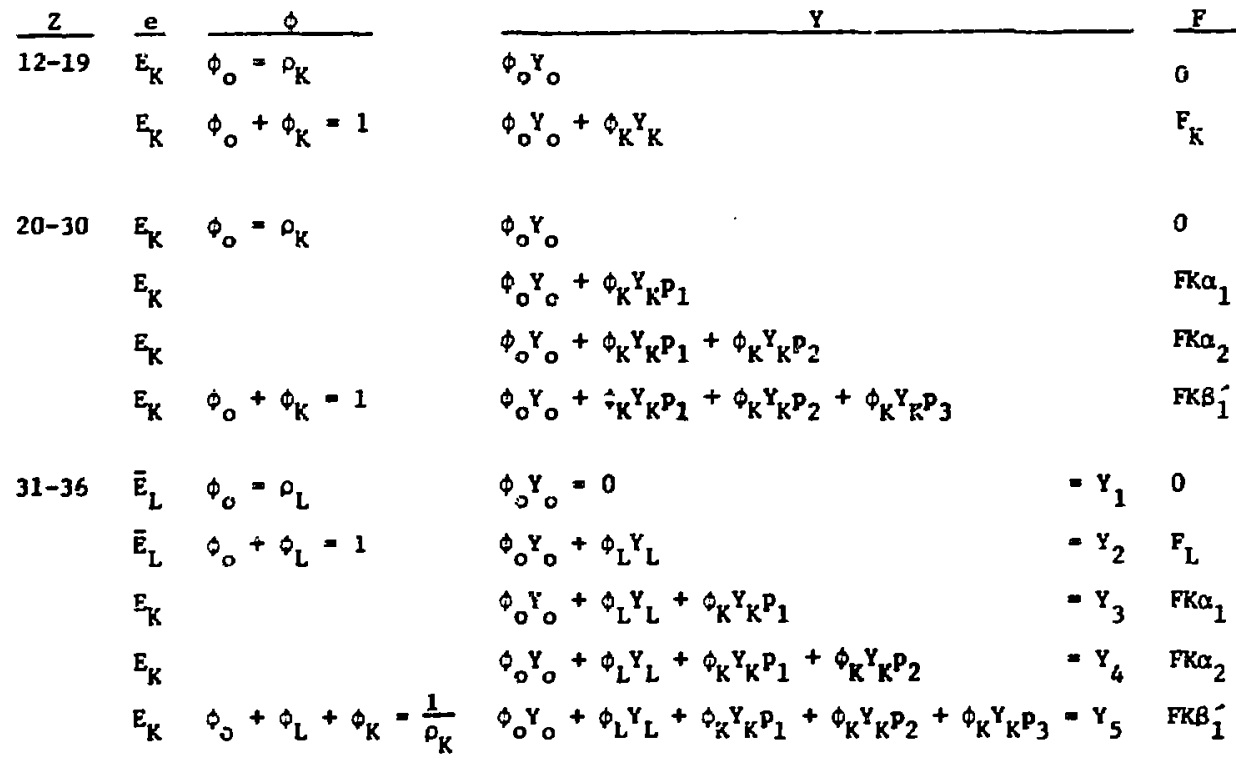

FiB. 5. Final $\phi, Y$ cabulation. 
the L3 or L2 subshell, and at the same time producIng a photon $K \alpha_{1}$, or $K \alpha_{2}$. If we denote by $Q_{L 3 K}$, $Q_{\text {L2K }}$ the chances of the latter photons escaping the atom (as fluorescence), then clearly, as part of the R-yield,

$$
P_{L 3 K} Q_{L 3 K}+P_{L 2 K} Q_{L 2 K}=Y_{K}^{P_{1}}+Y_{K} P_{2}
$$

in our previous notation.

But, regarding the L3, L2 subshells as separate entities, we may expect (secondary) L3 or L2 fluorescence with yield probabilities $Y_{L 3}, Y_{L 2}$, due to the now existing vacancles created by $L+K$ transition. Hence, the probability of this secondary L-fluorescence is glven by

$$
P^{\prime \prime}=\frac{\Phi_{K}}{\Sigma} \cdot\left(P_{L 3 K} Y_{L 3}+P_{L 2 K} Y_{L 2}\right) \text {. }
$$

Apparently none of the probabilities in parentheses are known. Making the assumption

$$
Y_{L 3}=Y_{L 2}=Y_{L 1}=Y_{L} / 3
$$

(5) becomes

$$
P^{\prime \prime}=\frac{\phi_{K}}{\sum}\left(P_{L 3 K}+P_{L 2 K}\right) Y_{L} / 3
$$

But from (7) and (4) we see that, for the secondary L-fluorescence,

$$
\mathrm{P}^{\prime \prime}>\frac{\phi_{\mathrm{K}}}{\Sigma}\left(\mathrm{Y}_{\mathrm{K}} \mathrm{P}_{1}+\mathrm{Y}_{\mathrm{K}} \mathrm{P}_{2}\right) \mathrm{Y}_{\mathrm{L}} / 3 \equiv \mathrm{P}^{*}
$$

and even $P^{\star}$ may exceed the primary probability $P^{\prime}$. For example, one finds from the appended tables (for $\mathrm{z}=79$ ),

$$
\begin{aligned}
\mathrm{P}^{\prime} & =\frac{.282}{4.859}=.058<\mathrm{P}^{\star}=\frac{3.213-.282}{4.859} \cdot \frac{.282}{.759} \cdot \frac{1}{3} \\
& \equiv .075<\mathrm{P}^{\prime \prime} .
\end{aligned}
$$

Thus, it would appear that secondary L-fluorescence should be included for the sake of consistency and accuracy.

Guided by the relation (8), we therefore make the following presumably inadequate provision in the revised code. In case of a p.e. event, on $2>31$ at $\mathrm{E}>\mathrm{E}_{\mathrm{K}}$, which is followed by $\mathrm{Ka}_{1}$ or $\mathrm{Ka}_{2}$ fluores- cence, we assume secondary L3 or L2 fluorescence emitted, each with probability $Y_{L} / 3$, and take $F_{L}$ as the fluorescent cnergy in elther case.

We include in Pig, 6 a flow diagram for the fluorescence subroutine. The only change from the previous one is the by-pass FS for banking secondary flu orescence, and the Bank emit from (N).

\section{REFERENCES}

1. E. D. Cashwe1I, J. R. Neergaard, C. J. Everett, R. G. Schrandt, H. M. Taylor, G. D. Turner, "Monte Car1o Codes: MCG and MCP," Los Alaws Scientific Laboratory report LA-5157-MS (Narch 1973).

2. E. Storm, H. I. Igrael, "Photon Cross Sections from $1 \mathrm{keV}$ to $100 \mathrm{HeV}$ for Elements ? = 1 to $2=100$," Nuclear Data Tables 7. 56;-688 (1970).

3. E. Storm, H. I. Israe1, "Photon Cross Sections from .001 to $100 \mathrm{MeV}$ for Elements 1 through 1C0," Log Alamog Scientific Laboratory report LA-3753 (1967).

4. R. H. Fink, R. C. Jopson, H. Mark, C, D. Swift, "Atomic Fluorescence Ylelds," Lawrence Radiation Laboratory report UCRL-14327 (1965).

5. C. R. Marotta, "Updated LIbrary Tape for Photran," A1r Fcxce Heapons Laboratory technical report AFUL-TR-67-11 (1967).

6. A. H. Wapstra et a1, Nuclear Spectrometry Tables, Interscience Publ1shers Inc., N.Y. (1959). 


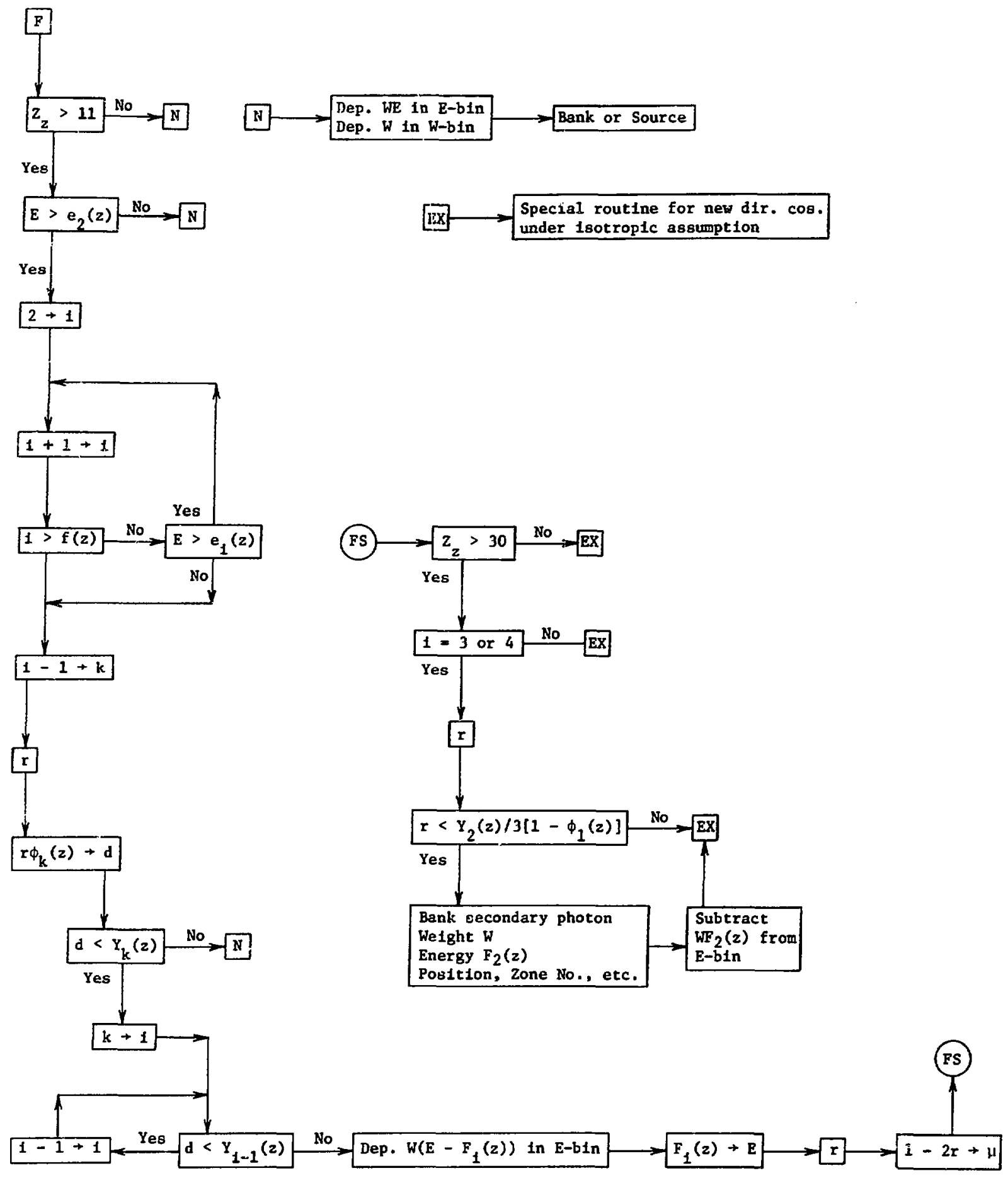

F1g. 6. Flow dlagram. 
TABLE I

MCP CODE FLUORESCENCE UPDATE

TABLE I (Cont ${ }^{2} d$ )

\begin{tabular}{|c|c|c|c|c|c|}
\hline$\underline{z}$ & $\underline{e}$ & $\phi$ & $Y$ & $\mathbf{F}$ & $\underline{\mathbf{f}}$ \\
\hline $\begin{array}{l}12 \\
\mathrm{Mg} \\
\end{array}$ & $\begin{array}{r}.001305 \\
.001305 \\
\end{array}$ & $\begin{array}{l}0.0784 \\
1.0 \\
\end{array}$ & $\begin{array}{l}0.0 \\
0.0258 \\
\end{array}$ & $\begin{array}{l}0.0 \\
0.001255 \\
\end{array}$ & 2. \\
\hline $\begin{array}{l}13 \\
\text { Al } \\
\end{array}$ & $\begin{array}{r}.001560 \\
.001560 \\
\end{array}$ & $\begin{array}{l}0.0839 \\
1.0 \\
\end{array}$ & $\begin{array}{l}0.0 \\
0.0347 \\
\end{array}$ & $\begin{array}{l}0.0 \\
0.001487 \\
\end{array}$ & 2 \\
\hline $\begin{array}{l}14 \\
\text { Si } \\
\end{array}$ & $\begin{array}{r}.001839 \\
.001839 \\
\end{array}$ & $\begin{array}{l}0.0888 \\
1.0 \\
\end{array}$ & $\begin{array}{l}0.0 \\
0.0465 \\
\end{array}$ & $\begin{array}{l}0.0 \\
0.001739 \\
\end{array}$ & 2 \\
\hline $\begin{array}{l}15 \\
\mathrm{P} \\
\end{array}$ & $\begin{array}{r}.002144 \\
.002144 \\
\end{array}$ & $\begin{array}{l}0.0927 \\
1.0 \\
\end{array}$ & $\begin{array}{l}0.0 \\
0.0599 \\
\end{array}$ & $\begin{array}{l}0.0 \\
0.002014 \\
\end{array}$ & 2 \\
\hline $\begin{array}{l}16 \\
\text { S }\end{array}$ & $\begin{array}{r}.002472 \\
.002472 \\
\end{array}$ & $\begin{array}{l}0.0966 \\
1.0 \\
\end{array}$ & $\begin{array}{l}0.0 \\
0.0732 \\
\end{array}$ & $\begin{array}{l}0.0 \\
0.002307 \\
\end{array}$ & 2 \\
\hline $\begin{array}{l}17 \\
\mathrm{C} 1 \\
\end{array}$ & $\begin{array}{r}.002824 \\
.002824 \\
\end{array}$ & $\begin{array}{l}0.1000 \\
1.0 \\
\end{array}$ & $\begin{array}{l}0.0 \\
0.0873 \\
\end{array}$ & $\begin{array}{l}0.0 \\
0.002622 \\
\end{array}$ & 2 \\
\hline $\begin{array}{l}18 \\
\text { Ar }\end{array}$ & $\begin{array}{r}.003203 \\
.003203 \\
\end{array}$ & $\begin{array}{l}0.104 \\
1.0 \\
\end{array}$ & $\begin{array}{l}0.0 \\
0.1066 \\
\end{array}$ & $\begin{array}{l}0.0 \\
0.002957 \\
\end{array}$ & 2 \\
\hline $\begin{array}{l}19 \\
\mathrm{~K} \\
\end{array}$ & $\begin{array}{r}.003607 \\
.003607 \\
\end{array}$ & $\begin{array}{l}0.108 \\
1.0 \\
\end{array}$ & $\begin{array}{l}0.0 \\
0.1267 \\
\end{array}$ & $\begin{array}{l}0.0 \\
0.003312 \\
\end{array}$ & 2 \\
\hline $\begin{array}{l}20 \\
\mathrm{Ca}\end{array}$ & $\begin{array}{l}.004037 \\
.004037 \\
.004037 \\
.004037 \\
\end{array}$ & $\begin{array}{l}0.110 \\
0.644 \\
0.915 \\
1.0 \\
\end{array}$ & $\begin{array}{l}0.0 \\
0.0892 \\
0.1345 \\
0.1487 \\
\end{array}$ & $\begin{array}{l}0.0 \\
0.003691 \\
0.003687 \\
0.004012 \\
\end{array}$ & 4 \\
\hline $\begin{array}{l}21 \\
S c\end{array}$ & $\begin{array}{l}.004491 \\
.004491 \\
.004491 \\
.004491 \\
\end{array}$ & $\begin{array}{l}0.113 \\
0.643 \\
0.913 \\
1.0 \\
\end{array}$ & $\begin{array}{l}0.0 \\
0.1007 \\
0.1520 \\
0.1687 \\
\end{array}$ & $\begin{array}{l}0.0 \\
0.004090 \\
0.004085 \\
0.004459 \\
\end{array}$ & 4 \\
\hline $\begin{array}{l}22 \\
T 1\end{array}$ & $\begin{array}{l}.004966 \\
.004966 \\
.004966 \\
.004966 \\
\end{array}$ & $\begin{array}{l}0.116 \\
0.642 \\
0.910 \\
1.0 \\
\end{array}$ & $\begin{array}{l}0.0 \\
0.1162 \\
0.1754 \\
0.1953 \\
\end{array}$ & $\begin{array}{l}0.0 \\
0.004510 \\
0.004504 \\
0.004931 \\
\end{array}$ & 4 \\
\hline $\begin{array}{l}23 \\
v\end{array}$ & $\begin{array}{l}.005465 \\
.005465 \\
.005465 \\
.005465 \\
\end{array}$ & $\begin{array}{l}0.117 \\
0.642 \\
0.910 \\
1.0 \\
\end{array}$ & $\begin{array}{l}0.0 \\
0.1297 \\
0.1959 \\
0.2184 \\
\end{array}$ & $\begin{array}{l}0.0 \\
0.004952 \\
0.004944 \\
0.005427 \\
\end{array}$ & 4 \\
\hline $\begin{array}{l}24 \\
\mathrm{Cr}\end{array}$ & $\begin{array}{r}.005989 \\
.005989 \\
.005989 \\
.005989 \\
\end{array}$ & $\begin{array}{l}0.120 \\
0.642 \\
0.908 \\
1.0 \\
\end{array}$ & $\begin{array}{l}0.0 \\
0.1441 \\
0.2175 \\
0.2429 \\
\end{array}$ & $\begin{array}{l}0.0 \\
0.005414 \\
0.005405 \\
0.005947 \\
\end{array}$ & 4 \\
\hline $\begin{array}{l}25 \\
M n\end{array}$ & $\begin{array}{l}.006539 \\
.006539 \\
.006539 \\
.006539 \\
\end{array}$ & $\begin{array}{l}0.122 \\
0.642 \\
0.907 \\
1.0 \\
\end{array}$ & $\begin{array}{l}0.0 \\
0.1544 \\
0.2331 \\
0.2607 \\
\end{array}$ & $\begin{array}{l}0.0 \\
0.005899 \\
0.005888 \\
0.006492 \\
\end{array}$ & 4 \\
\hline $\begin{array}{l}26 \\
\mathrm{Fe}\end{array}$ & $\begin{array}{l}.007112 \\
.007112 \\
.007112 \\
.007112 \\
\end{array}$ & $\begin{array}{l}0.124 \\
0.642 \\
0.907 \\
1.0 \\
\end{array}$ & $\begin{array}{l}0.0 \\
0.1673 \\
0.2529 \\
0.2833 \\
\end{array}$ & $\begin{array}{l}0.0 \\
0.006404 \\
0.006391 \\
0.007059 \\
\end{array}$ & 4 \\
\hline $\begin{array}{l}27 \\
\text { Co }\end{array}$ & $\begin{array}{r}.007709 \\
.007709 \\
.007709 \\
.007709 \\
\end{array}$ & $\begin{array}{l}0.126 \\
0.643 \\
0.907 \\
1.0 \\
\end{array}$ & $\begin{array}{l}0.0 \\
0.1810 \\
0.2734 \\
0.3063 \\
\end{array}$ & $\begin{array}{l}0.0 \\
0.006930 \\
0.006915 \\
0.007649 \\
\end{array}$ & 4 \\
\hline
\end{tabular}

\begin{tabular}{|c|c|c|c|c|c|}
\hline$\underline{z}$ & e & $\Phi$ & $Y$ & F & $\underline{\mathbf{f}}$ \\
\hline $\begin{array}{l}28 \\
\mathrm{~N} i\end{array}$ & $\begin{array}{l}.008332 \\
.008332 \\
.008332 \\
.008332 \\
\end{array}$ & $\begin{array}{l}0.127 \\
0.642 \\
0.906 \\
? .0 \\
\end{array}$ & $\begin{array}{l}0.0 \\
0.1942 \\
0.2937 \\
0.3291 \\
\end{array}$ & $\begin{array}{l}0.0 \\
0.007478 \\
0.007461 \\
0.008265 \\
\end{array}$ & 4 \\
\hline $\begin{array}{l}29 \\
\mathrm{Cu}\end{array}$ & $\begin{array}{r}.008981 \\
.008981 \\
.008981 \\
.008981 \\
\end{array}$ & $\begin{array}{l}0.129 \\
0.642 \\
0.905 \\
1.0 \\
\end{array}$ & $\begin{array}{l}0.0 \\
0.2083 \\
0.3151 \\
0.3537 \\
\end{array}$ & $\begin{array}{l}0.0 \\
0.008048 \\
0.008028 \\
0.008907 \\
\end{array}$ & 4 \\
\hline $\begin{array}{l}30 \\
\mathrm{Zn}\end{array}$ & $\begin{array}{r}.009659 \\
.009659 \\
.009659 \\
.009659 \\
\end{array}$ & $\begin{array}{l}0.130 \\
0.642 \\
0.905 \\
1.0 \\
\end{array}$ & $\begin{array}{l}0.0 \\
0.2217 \\
0.3356 \\
0.3772 \\
\end{array}$ & $\begin{array}{l}0.0 \\
0.008639 \\
0.008616 \\
0.009572 \\
\end{array}$ & 4 \\
\hline $\begin{array}{l}31 \\
\text { Ga }\end{array}$ & $\begin{array}{r}.001186 \\
.001186 \\
.010367 \\
.010367 \\
.010367 \\
\end{array}$ & $\begin{array}{l}0.118 \\
1.0 \\
4.861 \\
6.844 \\
7.588 \\
\end{array}$ & $\begin{array}{l}0.0 \\
0.0088 \\
1.808 \\
2.732 \\
3.079 \\
\end{array}$ & $\begin{array}{l}0.0 \\
0.001129 \\
0.009252 \\
0.009225 \\
0.010263 \\
\end{array}$ & 5 \\
\hline $\begin{array}{l}32 \\
\text { Ge }\end{array}$ & $\begin{array}{r}.001293 \\
.001293 \\
.011104 \\
.011104 \\
.011104 \\
\end{array}$ & $\begin{array}{l}0.125 \\
1.0 \\
4.814 \\
6.780 \\
7.531 \\
\end{array}$ & $\begin{array}{l}0.0 \\
0.0140 \\
1.921 \\
2.904 \\
3.280 \\
\end{array}$ & $\begin{array}{l}0.0 \\
0.001221 \\
0.009887 \\
0.009856 \\
0.010981 \\
\end{array}$ & 5 \\
\hline $\begin{array}{l}33 \\
\text { As }\end{array}$ & $\begin{array}{r}.001404 \\
.001404 \\
.011867 \\
.011867 \\
.011867 \\
\end{array}$ & $\begin{array}{l}0.129 \\
1.0 \\
4.754 \\
6.689 \\
7.450 \\
\end{array}$ & $\begin{array}{l}0.0 \\
0.0183 \\
2.015 \\
3.044 \\
3.451 \\
\end{array}$ & $\begin{array}{l}0.0 \\
0.001317 \\
0.010544 \\
0.020508 \\
0.011725 \\
\end{array}$ & 5 \\
\hline $\begin{array}{l}34 \\
\text { Se }\end{array}$ & $\begin{array}{l}.001520 \\
.001520 \\
.012658 \\
.012658 \\
.012658 \\
\end{array}$ & $\begin{array}{l}0.137 \\
1.0 \\
4.693 \\
6.603 \\
7.367 \\
\end{array}$ & $\begin{array}{l}0.0 \\
0.0233 \\
2.128 \\
3.217 \\
3.653 \\
\end{array}$ & $\begin{array}{l}0.0 \\
0.001416 \\
0.011224 \\
0.011183 \\
0.012495 \\
\end{array}$ & 5 \\
\hline $\begin{array}{l}35 \\
\text { Br }\end{array}$ & $\begin{array}{r}.001643 \\
.001643 \\
.013474 \\
.013474 \\
.013474 \\
\end{array}$ & $\begin{array}{l}0.141 \\
1.0 \\
4.624 \\
6.495 \\
7.259 \\
\end{array}$ & $\begin{array}{l}0.0 \\
0.0275 \\
2.192 \\
3.309 \\
3.765 \\
\end{array}$ & $\begin{array}{l}0.0 \\
0.001519 \\
0.011923 \\
0.011877 \\
0.013288 \\
\end{array}$ & 5 \\
\hline $\begin{array}{l}36 \\
\mathrm{Kr}\end{array}$ & $\begin{array}{r}.001774 \\
.001774 \\
.014323 \\
.014323 \\
.014323 \\
\end{array}$ & $\begin{array}{l}0.147 \\
1.0 \\
4.570 \\
6.420 \\
7.187 \\
\end{array}$ & $\begin{array}{l}0.0 \\
0.0333 \\
2.282 \\
3.448 \\
3.931 \\
\end{array}$ & $\begin{array}{l}0.0 \\
0.001631 \\
0.012648 \\
0.012596 \\
0.014110 \\
\end{array}$ & 5 \\
\hline $\begin{array}{l}37 \\
\mathrm{Rb}\end{array}$ & $\begin{array}{l}.001911 \\
.001911 \\
.015200 \\
.015200 \\
.015200 \\
.015200 \\
\end{array}$ & $\begin{array}{l}0.152 \\
1.0 \\
4.452 \\
6.245 \\
6.999 \\
7.078 \\
\end{array}$ & $\begin{array}{l}0.0 \\
0.0373 \\
2.312 \\
3.494 \\
3.991 \\
4.043 \\
\end{array}$ & $\begin{array}{l}0.0 \\
0.001745 \\
0.013395 \\
0.013337 \\
0.014958 \\
0.015185 \\
\end{array}$ & 6 \\
\hline $\begin{array}{l}38 \\
\mathrm{Sr}\end{array}$ & $\begin{array}{l}.002054 \\
.002054 \\
.016105 \\
.016105 \\
.016105 \\
.016105\end{array}$ & $\begin{array}{l}0.155 \\
1.0 \\
4.380 \\
6.139 \\
6.893 \\
6.983 \\
\end{array}$ & $\begin{array}{l}0.0 \\
0.04 j .4 \\
2.356 \\
3.561 \\
4.077 \\
4.138 \\
\end{array}$ & $\begin{array}{l}0.0 \\
0.001864 \\
0.014165 \\
0.014098 \\
0.015832 \\
0.016085\end{array}$ & 6 \\
\hline
\end{tabular}


TABLE I (Cont'd)

\begin{tabular}{|c|c|c|c|c|c|}
\hline$\underline{2}$ & e & $\phi$ & $\mathbf{r}$ & $\boldsymbol{F}$ & $\underline{f}$ \\
\hline $\begin{array}{l}39 \\
Y\end{array}$ & $\begin{array}{r}.002202 \\
.002202 \\
.017038 \\
.017038 \\
.017038 \\
.017038 \\
\end{array}$ & $\begin{array}{l}0.161 \\
1.0 \\
4.327 \\
6.058 \\
6.814 \\
6.908 \\
\end{array}$ & $\begin{array}{l}0.0 \\
0.0461 \\
2.402 \\
3.628 \\
4.164 \\
4.231 \\
\end{array}$ & $\begin{array}{l}0.0 \\
0.001987 \\
0.014959 \\
0.014883 \\
0.016735 \\
0.017013 \\
\end{array}$ & 6 \\
\hline $\begin{array}{l}40 \\
\mathrm{Zr}\end{array}$ & $\begin{array}{r}.002354 \\
.002354 \\
.017998 \\
.017998 \\
.017998 \\
.017998 \\
\end{array}$ & $\begin{array}{l}0.166 \\
1.0 \\
4.280 \\
5.996 \\
6.757 \\
6.857 \\
\end{array}$ & $\begin{array}{l}0.0 \\
0.0500 \\
2.444 \\
3.697 \\
4.253 \\
4.326 \\
\end{array}$ & $\begin{array}{l}0.0 \\
0.002113 \\
0.015775 \\
0.015691 \\
0.017663 \\
0.017969 \\
\end{array}$ & 6 \\
\hline $\begin{array}{l}41 \\
\mathrm{Nb}\end{array}$ & $\begin{array}{r}.002511 \\
.002511 \\
.018986 \\
.018986 \\
.018986 \\
.018986 \\
\end{array}$ & $\begin{array}{l}0.171 \\
1.0 \\
4.217 \\
5.901 \\
6.662 \\
6.766 \\
\end{array}$ & $\begin{array}{l}0.0 \\
0.0547 \\
2.458 \\
3.716 \\
4.285 \\
4.363 \\
\end{array}$ & $\begin{array}{l}0.0 \\
0.002244 \\
0.016616 \\
0.016522 \\
0.018620 \\
0.018954 \\
\end{array}$ & 6 \\
\hline $\begin{array}{l}42 \\
\text { Mo }\end{array}$ & $\begin{array}{l}.002671 \\
.002671 \\
.020000 \\
.020000 \\
.020000 \\
.020000 \\
\end{array}$ & $\begin{array}{l}0.175 \\
1.0 \\
4.193 \\
5.873 \\
6.644 \\
6.753 \\
\end{array}$ & $\begin{array}{l}0.0 \\
0.0594 \\
2.495 \\
3.777 \\
4.365 \\
4.448 \\
\end{array}$ & $\begin{array}{l}0.0 \\
0.002378 \\
0.017479 \\
0.017375 \\
0.019602 \\
0.019965 \\
\end{array}$ & $\mathbf{6}$ \\
\hline $\begin{array}{c}43 \\
\text { Tc } \\
\text { * }\end{array}$ & $\begin{array}{l}.002838 \\
.002838 \\
.021044 \\
.021044 \\
.021044 \\
.021044 \\
\end{array}$ & $\begin{array}{l}0.178 \\
1.0 \\
4.134 \\
5.783 \\
6.554 \\
6.667 \\
\end{array}$ & $\begin{array}{l}0.0 \\
0.0641 \\
2.502 \\
3.785 \\
4.335 \\
4.473 \\
\end{array}$ & $\begin{array}{l}0.0 \\
0.002517 \\
0.018367 \\
0.018251 \\
0.020612 \\
0.021006 \\
\end{array}$ & 6 \\
\hline $\begin{array}{l}44 \\
\text { Ru }\end{array}$ & $\begin{array}{l}.003010 \\
.003010 \\
.022117 \\
.022117 \\
.022117 \\
.022117 \\
\end{array}$ & $\begin{array}{l}0.183 \\
1.0 \\
4.075 \\
5.696 \\
6.459 \\
6.570 \\
\end{array}$ & $\begin{array}{l}0.0 \\
0.0678 \\
2.510 \\
3.797 \\
4.403 \\
4.491 \\
\end{array}$ & $\begin{array}{l}0.0 \\
0.002669 \\
0.019279 \\
0.019150 \\
0.021649 \\
0.022075 \\
\end{array}$ & 6 \\
\hline $\begin{array}{l}45 \\
\text { Rh }\end{array}$ & $\begin{array}{l}.003187 \\
.003187 \\
.023220 \\
.023220 \\
.023220 \\
.023220 \\
\end{array}$ & $\begin{array}{l}0.184 \\
1.0 \\
4.018 \\
5.615 \\
6.372 \\
6.487 \\
\end{array}$ & $\begin{array}{l}0.0 \\
0.0726 \\
2.506 \\
3.793 \\
4.403 \\
4.496 \\
\end{array}$ & $\begin{array}{l}0.0 \\
0.002820 \\
0.020216 \\
0.020074 \\
0.022716 \\
0.023173 \\
\end{array}$ & 6 \\
\hline $\begin{array}{l}46 \\
\text { Pd }\end{array}$ & $\begin{array}{l}.003370 \\
.003370 \\
.024350 \\
.024350 \\
.024350 \\
.024350 \\
\end{array}$ & $\begin{array}{l}0.188 \\
1.0 \\
3.958 \\
5.523 \\
6.273 \\
6.397 \\
\end{array}$ & $\begin{array}{l}0.0 \\
0.0771 \\
2.500 \\
3.782 \\
4.396 \\
4.498 \\
\end{array}$ & $\begin{array}{l}0.0 \\
0.002976 \\
0.021176 \\
0.021020 \\
0.023809 \\
0.024298 \\
\end{array}$ & 6 \\
\hline $\begin{array}{l}47 \\
\mathrm{Ag}\end{array}$ & $\begin{array}{l}.003560 \\
.003560 \\
.025514 \\
.025514 \\
.025514 \\
.025514\end{array}$ & $\begin{array}{l}0.190 \\
1.0 \\
3.927 \\
5.482 \\
6.233 \\
6.362 \\
\end{array}$ & $\begin{array}{l}0.0 \\
0.0810 \\
2.510 \\
3.801 \\
4.424 \\
4.531 \\
\end{array}$ & $\begin{array}{l}0.0 \\
0.003136 \\
0.022163 \\
0.021990 \\
0.024933 \\
0.025456\end{array}$ & 6 \\
\hline
\end{tabular}

TABLE I (Cont'd)

\begin{tabular}{|c|c|c|c|c|c|}
\hline$\underline{2}$ & e & $\phi$ & $\mathbf{Y}$ & F & $\underline{\mathbf{f}}$ \\
\hline $\begin{array}{l}48 \\
\text { Cd }\end{array}$ & $\begin{array}{l}.003761 \\
.003761 \\
.026 / 11 \\
.026711 \\
.026711 \\
.026711 \\
\end{array}$ & $\begin{array}{l}0.192 \\
1.0 \\
3.911 \\
5.463 \\
6.223 \\
6.351 \\
\end{array}$ & $\begin{array}{l}0.0 \\
0.0856 \\
2.534 \\
3.839 \\
4.4 i 8 \\
4.586 \\
\end{array}$ & $\begin{array}{l}0.0 \\
0.003301 \\
0.023174 \\
0.022984 \\
0.026084 \\
0.026646 \\
\end{array}$ & 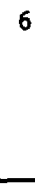 \\
\hline $\begin{array}{l}49 \\
\text { In }\end{array}$ & $\begin{array}{r}.003969 \\
.003969 \\
.027940 \\
.027940 \\
.027940 \\
.027940 \\
\end{array}$ & $\begin{array}{l}0.195 \\
1.0 \\
3.880 \\
5.413 \\
6.171 \\
6.304 \\
\end{array}$ & $\begin{array}{l}0.0 \\
0.0902 \\
2.538 \\
3.841 \\
4.485 \\
4.598 \\
\end{array}$ & $\begin{array}{l}0.0 \\
0.003472 \\
0.024210 \\
0.024002 \\
0.027264 \\
0.027866 \\
\end{array}$ & 6 \\
\hline $\begin{array}{l}50 \\
\text { Sn }\end{array}$ & $\begin{array}{r}.004183 \\
.004183 \\
.029200 \\
.029200 \\
.029200 \\
.029200 \\
\end{array}$ & $\begin{array}{l}0.198 \\
1.0 \\
3.820 \\
5.326 \\
6.077 \\
6.213 \\
\end{array}$ & $\begin{array}{l}0.0 \\
0.0946 \\
2.529 \\
3.829 \\
4.477 \\
4.594 \\
\end{array}$ & $\begin{array}{l}0.0 \\
0.003647 \\
0.025271 \\
0.025044 \\
0.028472 \\
0.029114 \\
\end{array}$ & 6 \\
\hline $\begin{array}{l}51 \\
\text { Sb }\end{array}$ & $\begin{array}{l}.004404 \\
.004404 \\
.030491 \\
.030491 \\
.030491 \\
.030491 \\
\end{array}$ & $\begin{array}{l}0.200 \\
1.0 \\
3.777 \\
5.266 \\
6.013 \\
6.152 \\
\end{array}$ & $\begin{array}{l}0.0 \\
0.0984 \\
2.508 \\
3.800 \\
4.448 \\
4.569 \\
\end{array}$ & $\begin{array}{l}0.0 \\
0.003828 \\
0.026359 \\
0.026110 \\
0.029710 \\
0.030392 \\
\end{array}$ & 6 \\
\hline $\begin{array}{l}52 \\
\text { Te }\end{array}$ & $\begin{array}{r}.004631 \\
.004631 \\
.031814 \\
.031814 \\
.031814 \\
.031814 \\
\end{array}$ & $\begin{array}{l}0.202 \\
1.0 \\
3.744 \\
5.221 \\
5.967 \\
6.110 \\
\end{array}$ & $\begin{array}{l}0.0 \\
0.1029 \\
2.501 \\
3.792 \\
4.444 \\
4.569 \\
\end{array}$ & $\begin{array}{l}0.0 \\
0.004014 \\
0.027473 \\
0.027202 \\
0.030978 \\
0.031701 \\
\end{array}$ & 6 \\
\hline 53 & $\begin{array}{r}.004866 \\
.004866 \\
.033170 \\
.033170 \\
.033170 \\
.033170 \\
\end{array}$ & $\begin{array}{l}0.204 \\
1.0 \\
3.720 \\
5.186 \\
5.932 \\
6.074 \\
\end{array}$ & $\begin{array}{l}0.0 \\
0.1067 \\
2.498 \\
3.787 \\
4.443 \\
4.568 \\
\end{array}$ & $\begin{array}{l}0.0 \\
0.004206 \\
i .028613 \\
0.028318 \\
0.032276 \\
0.033041 \\
\end{array}$ & 6 \\
\hline $\begin{array}{l}54 \\
\mathrm{Xe}\end{array}$ & $\begin{array}{l}.005110 \\
.005110 \\
.034561 \\
.034561 \\
.034561 \\
.034561 \\
\end{array}$ & $\begin{array}{l}0.205 \\
1.0 \\
3.679 \\
5.129 \\
5.872 \\
6.017 \\
\end{array}$ & $\begin{array}{l}0.0 \\
0.1113 \\
2.479 \\
3.761 \\
4.418 \\
4.546 \\
\end{array}$ & $\begin{array}{l}0.0 \\
0.004602 \\
0.0297 \\
0.02945 \\
0.03360 \\
0.034415 \\
\end{array}$ & 6 \\
\hline $\begin{array}{l}55 \\
\mathrm{Cs}\end{array}$ & $\begin{array}{r}.005362 \\
.005362 \\
.035985 \\
.035985 \\
.035985 \\
.035985 \\
\end{array}$ & $\begin{array}{l}0.206 \\
1.0 \\
3.637 \\
5.062 \\
5.799 \\
5.947 \\
\end{array}$ & $\begin{array}{l}0.0 \\
0.1159 \\
2.463 \\
3.731 \\
4.387 \\
4.519 \\
\end{array}$ & $\begin{array}{l}0.0 \\
0.004603 \\
0.030973 \\
0.030625 \\
0.034965 \\
0.035820 \\
\end{array}$ & 6 \\
\hline $\begin{array}{l}56 \\
\mathrm{Ba}\end{array}$ & $\begin{array}{l}.005619 \\
.005619 \\
.037441 \\
.037441 \\
.037441 \\
.037441 \\
\end{array}$ & $\begin{array}{l}0.208 \\
1.0 \\
3.602 \\
5.013 \\
5.748 \\
5.900 \\
\end{array}$ & $\begin{array}{l}0.0 \\
0.1196 \\
2.446 \\
3.707 \\
4.364 \\
4.500 \\
\end{array}$ & $\begin{array}{l}0.0 \\
0.004811 \\
0.032194 \\
0.031818 \\
0.036356 \\
0.037257 \\
\end{array}$ & 6 \\
\hline
\end{tabular}


TABLE I (Cont'd)

\begin{tabular}{|c|c|c|c|c|c|}
\hline$\underline{z}$ & e & $\phi$ & $\mathbf{Y}$ & $\underline{F}$ & $\underline{E}$ \\
\hline $\begin{array}{l}57 \\
\text { La }\end{array}$ & $\begin{array}{l}.005880 \\
.005880 \\
.038925 \\
.038925 \\
.038925 \\
.038925 \\
\end{array}$ & $\begin{array}{l}0.209 \\
1.0 \\
3.551 \\
4.937 \\
5.659 \\
5.813 \\
\end{array}$ & $\begin{array}{l}0.0 \\
0.1250 \\
2.416 \\
3.661 \\
4.309 \\
4.447 \\
\end{array}$ & $\begin{array}{l}0.0 \\
0.005022 \\
0.033441 \\
0.033034 \\
0.037775 \\
0.038728 \\
\end{array}$ & 6 \\
\hline $\begin{array}{l}58 \\
\mathrm{Ce} \\
*\end{array}$ & $\begin{array}{l}.006145 \\
.006145 \\
.040443 \\
.040443 \\
.040443 \\
.040443 \\
\end{array}$ & $\begin{array}{l}0.211 \\
1.0 \\
3.532 \\
4.918 \\
5.642 \\
5.795 \\
\end{array}$ & $\begin{array}{l}0.0 \\
0.1278 \\
2.412 \\
3.662 \\
4.315 \\
4.453 \\
\end{array}$ & $\begin{array}{l}0.0 \\
C .005237 \\
0.034720 \\
0.034279 \\
0.039229 \\
0.040233 \\
\end{array}$ & 6 \\
\hline $\begin{array}{l}59 \\
\mathbf{P r} \\
\star\end{array}$ & $\begin{array}{l}.006413 \\
.006413 \\
.041991 \\
.041991 \\
.041991 \\
.041991 \\
\end{array}$ & $\begin{array}{l}0.211 \\
1.0 \\
3.491 \\
4.857 \\
5.575 \\
5.726 \\
\end{array}$ & $\begin{array}{l}0.0 \\
0.1333 \\
2.387 \\
3.623 \\
4.273 \\
4.410 \\
\end{array}$ & $\begin{array}{l}0.0 \\
0.005450 \\
0.036027 \\
0.035551 \\
0.040718 \\
0.041769 \\
\end{array}$ & 6 \\
\hline $\begin{array}{l}60 \\
\mathrm{Nd}\end{array}$ & $\begin{array}{l}.006686 \\
.006686 \\
.043569 \\
.043569 \\
.043569 \\
.043569 \\
\end{array}$ & $\begin{array}{l}0.213 \\
1.0 \\
3.461 \\
4.813 \\
5.529 \\
5.679 \\
\end{array}$ & $\begin{array}{l}0.0 \\
0.1362 \\
2.376 \\
3.606 \\
4.258 \\
4.394 \\
\end{array}$ & $\begin{array}{l}0.0 \\
0.005684 \\
0.037361 \\
0.036847 \\
0.042237 \\
0.043335 \\
\end{array}$ & 6 \\
\hline $\begin{array}{c}61 \\
\text { Pm } \\
\star\end{array}$ & $\begin{array}{l}.006967 \\
.006967 \\
.045184 \\
.045184 \\
.045184 \\
.045184 \\
\end{array}$ & $\begin{array}{l}0.215 \\
1.0 \\
3.452 \\
4.802 \\
5.517 \\
5.671 \\
\end{array}$ & $\begin{array}{l}0.0 \\
0.1413 \\
2.377 \\
3.608 \\
4.260 \\
4.400 \\
\end{array}$ & $\begin{array}{l}0.0 \\
0.005917 \\
0.038725 \\
0.038171 \\
0.043790 \\
0.044939 \\
\end{array}$ & 6 \\
\hline $\begin{array}{l}62 \\
\text { Sm } \\
\star\end{array}$ & $\begin{array}{l}.007255 \\
.007255 \\
.046834 \\
.046834 \\
.046834 \\
.046834 \\
\end{array}$ & $\begin{array}{l}0.216 \\
1.0 \\
3.410 \\
4.742 \\
5.452 \\
5.609 \\
\end{array}$ & $\begin{array}{l}0.0 \\
0.1450 \\
2.353 \\
3.573 \\
4.223 \\
4.367 \\
\end{array}$ & $\begin{array}{l}0.0 \\
0.006154 \\
0.040118 \\
0.039522 \\
0.045376 \\
0.046579 \\
\end{array}$ & 6 \\
\hline $\begin{array}{l}63 \\
\mathrm{Eu}\end{array}$ & $\begin{array}{r}.007549 \\
.007549 \\
.048519 \\
.048519 \\
.048519 \\
.048519 \\
\end{array}$ & $\begin{array}{l}0.216 \\
1.0 \\
3.382 \\
4.701 \\
5.408 \\
5.563 \\
\end{array}$ & $\begin{array}{l}0.0 \\
0.1497 \\
2.341 \\
3.554 \\
4.204 \\
4.347 \\
\end{array}$ & $\begin{array}{l}0.0 \\
0.006396 \\
0.041542 \\
0.040901 \\
0.046995 \\
0.048253 \\
\end{array}$ & 6 \\
\hline $\begin{array}{c}64 \\
\text { Gd } \\
*\end{array}$ & $\begin{array}{l}.007849 \\
.007849 \\
.050239 \\
.050239 \\
.050239 \\
.050239 \\
\end{array}$ & $\begin{array}{l}0.219 \\
1.0 \\
3.360 \\
4.673 \\
5.375 \\
5.529 \\
\end{array}$ & $\begin{array}{l}0.0 \\
0.1539 \\
2.330 \\
3.541 \\
4.188 \\
4.330 \\
\end{array}$ & $\begin{array}{l}0.0 \\
0.006642 \\
0.042996 \\
0.042309 \\
0.048649 \\
0.049959 \\
\end{array}$ & 6 \\
\hline $\begin{array}{c}65 \\
\mathrm{~Tb} \\
\star\end{array}$ & $\begin{array}{l}.008158 \\
.008158 \\
.051996 \\
.051996 \\
.051996 \\
.051996\end{array}$ & $\begin{array}{l}0.220 \\
1.0 \\
3.329 \\
4.628 \\
5.327 \\
5.479 \\
\end{array}$ & $\begin{array}{l}0.0 \\
0.1576 \\
2.315 \\
3.518 \\
4.165 \\
4.306 \\
\end{array}$ & $\begin{array}{l}0.0 \\
0.006896 \\
0.044482 \\
0.043744 \\
0.050336 \\
0.051703 \\
\end{array}$ & 6 \\
\hline
\end{tabular}

IABLE I (Cont'd)

\begin{tabular}{|c|c|c|c|c|c|}
\hline$\underline{2}$ & e & $\phi$ & $I$ & $F$ & $E$ \\
\hline $\begin{array}{l}66 \\
D_{y}\end{array}$ & $\begin{array}{l}.008472 \\
.008472 \\
.053788 \\
.053788 \\
.053788 \\
.053788 \\
\end{array}$ & $\begin{array}{l}0.223 \\
1.0 \\
3.305 \\
4.595 \\
5.294 \\
5.450 \\
\end{array}$ & $\begin{array}{l}0.0 \\
0.2624 \\
2.303 \\
3.501 \\
4.150 \\
4.295 \\
\end{array}$ & $\begin{array}{l}0.0 \\
0.007155 \\
0.045998 \\
0.045208 \\
0.05205 \mathrm{~B} \\
0.0534 \mathrm{BZ} \\
\end{array}$ & \\
\hline $\begin{array}{l}67 \\
\text { Ho } \\
\star\end{array}$ & $\begin{array}{l}.008795 \\
.008795 \\
.055618 \\
.055618 \\
.055618 \\
.055618 \\
\end{array}$ & $\begin{array}{l}0.222 \\
1.0 \\
3.274 \\
4.554 \\
5.245 \\
5.399 \\
\end{array}$ & $\begin{array}{l}0.0 \\
0.1665 \\
2.283 \\
3.475 \\
4.118 \\
4.261 \\
\end{array}$ & $\begin{array}{l}0.0 \\
0.007420 \\
0.047546 \\
0.046700 \\
0.053817 \\
0.055297 \\
\end{array}$ & \\
\hline $\begin{array}{c}68 \\
\mathrm{Er} \\
\star\end{array}$ & $\begin{array}{l}.009125 \\
.009125 \\
.057486 \\
.057486 \\
.057486 \\
.057486 \\
\end{array}$ & $\begin{array}{l}0.223 \\
1.0 \\
3.249 \\
4.517 \\
5.206 \\
5.359 \\
\end{array}$ & $\begin{array}{l}0.0 \\
0.1709 \\
2.272 \\
3.456 \\
4.100 \\
4.243 \\
\end{array}$ & $\begin{array}{l}0.0 \\
0.007691 \\
0.049128 \\
0.048222 \\
0.055611 \\
0.057151 \\
\end{array}$ & \\
\hline $\begin{array}{l}69 \\
\mathrm{Tm}\end{array}$ & $\begin{array}{l}.009460 \\
.009460 \\
.059390 \\
.059390 \\
.059390 \\
.059390 \\
\end{array}$ & $\begin{array}{l}0.225 \\
1.0 \\
3.225 \\
4.482 \\
5.169 \\
5.320 \\
\end{array}$ & $\begin{array}{l}0.0 \\
0.1744 \\
2.259 \\
3.437 \\
4.081 \\
4.222 \\
\end{array}$ & $\begin{array}{l}0.0 \\
0.007966 \\
0.050742 \\
0.049773 \\
0.057441 \\
0.059039 \\
\end{array}$ & \\
\hline $\begin{array}{l}70 \\
\text { Yb } \\
\star\end{array}$ & $\begin{array}{r}.009803 \\
.009803 \\
.061332 \\
.061332 \\
.061332 \\
.061332 \\
\end{array}$ & $\begin{array}{l}0.225 \\
1.0 \\
3.196 \\
4.446 \\
5.131 \\
5.281 \\
\end{array}$ & $\begin{array}{l}0.0 \\
0.1782 \\
2.240 \\
3.414 \\
4.057 \\
4.198 \\
\end{array}$ & $\begin{array}{l}0.0 \\
0.08250 \\
0.052389 \\
0.051354 \\
0.059308 \\
0.060965 \\
\end{array}$ & 6 \\
\hline $\begin{array}{l}71 \\
\text { Lu } \\
\star\end{array}$ & $\begin{array}{l}.010156 \\
.020156 \\
.063316 \\
.063316 \\
.065316 \\
.063316 \\
\end{array}$ & $\begin{array}{l}0.227 \\
1.0 \\
3.159 \\
4.390 \\
5.065 \\
5.217 \\
\end{array}$ & $\begin{array}{l}0.0 \\
0.1832 \\
2.215 \\
3.373 \\
4.008 \\
4.151 \\
\end{array}$ & $\begin{array}{l}0.0 \\
0.008540 \\
0.054071 \\
0.052967 \\
0.061213 \\
0.062931 \\
\end{array}$ & 6 \\
\hline $\begin{array}{l}72 \\
\text { Hf }\end{array}$ & $\begin{array}{l}.010524 \\
.010524 \\
.065345 \\
.065345 \\
.065345 \\
.065345 \\
\end{array}$ & $\begin{array}{l}0.22 \% \\
1.0 \\
3.131 \\
4.349 \\
5.020 \\
5.170 \\
\end{array}$ & $\begin{array}{l}0.0 \\
0.1866 \\
2.194 \\
3.341 \\
3.973 \\
4.114 \\
\end{array}$ & $\begin{array}{l}0.0 \\
0.008839 \\
0.055785 \\
0.054606 \\
0.063153 \\
0.064942 \\
\end{array}$ & 6 \\
\hline $\begin{array}{l}73 \\
\text { Ta }\end{array}$ & $\begin{array}{l}.010899 \\
.010899 \\
.067416 \\
.067416 \\
.067416 \\
.067416 \\
\end{array}$ & $\begin{array}{l}0.229 \\
1.0 \\
3.110 \\
4.322 \\
4.988 \\
5.137 \\
\end{array}$ & $\begin{array}{l}0.0 \\
0.1912 \\
2.183 \\
3.327 \\
3.956 \\
4.097 \\
\end{array}$ & $\begin{array}{l}0.0 \\
0.009142 \\
0.037536 \\
0.056280 \\
0.065132 \\
0.066991 \\
\end{array}$ & 6 \\
\hline $\begin{array}{l}74 \\
W\end{array}$ & $\begin{array}{l}.011281 \\
.011281 \\
.069525 \\
.069525 \\
.069525 \\
.069525\end{array}$ & $\begin{array}{l}0.231 \\
1.0 \\
3.084 \\
4.284 \\
4.947 \\
5.094 \\
\end{array}$ & $\begin{array}{l}0.0 \\
0.2253 \\
2.196 \\
3.332 \\
3.958 \\
4.097\end{array}$ & $\begin{array}{l}0.0 \\
0.009454 \\
0.059321 \\
0.057994 \\
0.067147 \\
0.069078\end{array}$ & 6 \\
\hline
\end{tabular}


TABLE I (cone'd)

\begin{tabular}{|c|c|c|c|c|c|}
\hline$\underline{2}$ & $\mathbf{e}$ & $\theta$ & $y$ & $E$ & 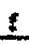 \\
\hline $\begin{array}{l}75 \\
\text { Re }\end{array}$ & $\begin{array}{l}.011673 \\
.011673 \\
.071676 \\
.071676 \\
.071676 \\
.072676 \\
\end{array}$ & $\begin{array}{l}0.232 \\
1.0 \\
3.050 \\
4.248 \\
4.909 \\
5.055 \\
\end{array}$ & $\begin{array}{l}0.0 \\
0.2627 \\
2.196 \\
3.322 \\
3.949 \\
4.087 \\
\end{array}$ & $\begin{array}{l}0.0 \\
0.009775 \\
0.061142 \\
0.059719 \\
0.069205 \\
0.071206\end{array}$ & 6 \\
\hline $\begin{array}{l}76 \\
03\end{array}$ & $\begin{array}{l}.022075 \\
.012075 \\
.073871 \\
.073871 \\
.073871 \\
.073871 \\
\end{array}$ & $\begin{array}{l}0.232 \\
1.0 \\
3.020 \\
9.294 \\
4.845 \\
4.993 \\
\end{array}$ & $\begin{array}{l}0.0 \\
0.2565 \\
2.173 \\
3.287 \\
3.905 \\
4.065 \\
\end{array}$ & $\begin{array}{l}0.0 \\
0.010103 \\
0.063000 \\
0.061486 \\
0.071306 \\
0.073377\end{array}$ & 6 \\
\hline $\begin{array}{l}77 \\
17\end{array}$ & $\begin{array}{l}.012486 \\
.012486 \\
.076111 \\
.076121 \\
.076211 \\
.076212 \\
\end{array}$ & $\begin{array}{l}0.235 \\
2.0 \\
2.999 \\
4.163 \\
4.812 \\
4.958 \\
\end{array}$ & $\begin{array}{l}0.0 \\
0.2670 \\
2.166 \\
3.272 \\
3.889 \\
4.028 \\
\end{array}$ & $\begin{array}{l}0.0 \\
0.010436 \\
0.064896 \\
0.063287 \\
0.073442 \\
0.075590 \\
\end{array}$ & 6 \\
\hline $\begin{array}{l}78 \\
9 t\end{array}$ & $\begin{array}{l}.012906 \\
.012906 \\
.078395 \\
.075395 \\
.078395 \\
.028995\end{array}$ & $\begin{array}{l}0.236 \\
1.0 \\
2.956 \\
4.114 \\
4.753 \\
4.397 \\
\end{array}$ & $\begin{array}{l}0.0 \\
0.2758 \\
2.146 \\
3.238 \\
3.846 \\
3.983 \\
\end{array}$ & $\begin{array}{l}0.0 \\
0.010775 \\
0.066531 \\
0.065122 \\
0.075824 \\
0.077046 \\
\end{array}$ & 6 \\
\hline $\begin{array}{l}79 \\
\text { A U }\end{array}$ & $\begin{array}{l}.013335 \\
.023135 \\
.080725 \\
.089725 \\
.080725 \\
.080725\end{array}$ & $\begin{array}{l}0.241 \\
1.0 \\
2.941 \\
4.079 \\
4.712 \\
4.859 \\
\end{array}$ & $\begin{array}{l}0.0 \\
0.2823 \\
2.130 \\
3.213 \\
3.816 \\
3.956 \\
\end{array}$ & $\begin{array}{l}0.0 \\
0.011125 \\
0.068806 \\
0.066991 \\
0.077868 \\
0.080146 \\
\end{array}$ & 6 \\
\hline $\begin{array}{l}80 \\
H_{6}\end{array}$ & $\begin{array}{l}.013770 \\
.013778 \\
.083102 \\
.083102 \\
.083107 \\
.083102 \\
\end{array}$ & $\begin{array}{l}0.240 \\
1.0 \\
2.909 \\
4.035 \\
4.660 \\
4.809 \\
\end{array}$ & $\begin{array}{l}0.0 \\
0.2503 \\
2.208 \\
3.182 \\
3.778 \\
3.920 \\
\end{array}$ & $\begin{array}{l}0.0 \\
0.011460 \\
0.070819 \\
0.068893 \\
0.080112 \\
0.082491 \\
\end{array}$ & 6 \\
\hline 81 & $\begin{array}{l}.014233 \\
.014233 \\
.085530 \\
.085530 \\
.055530 \\
.085530\end{array}$ & $\begin{array}{l}0.241 \\
1.0 \\
2.879 \\
3.992 \\
6.612 \\
4.258 \\
\end{array}$ & $\begin{array}{l}0.0 \\
0.2968 \\
2.090 \\
3.251 \\
3.742 \\
3.802 \\
\end{array}$ & $\begin{array}{l}0.0 \\
0.014015 \\
0.072874 \\
0.070833 \\
0.002422 \\
0.034883\end{array}$ & 6 \\
\hline 82 & $\begin{array}{l}.014599 \\
.014659 \\
.088006 \\
.088004 \\
.088004 \\
.088004 \\
\end{array}$ & $\begin{array}{l}0.242 \\
2.0 \\
2.854 \\
3.957 \\
4.570 \\
4.715 \\
\end{array}$ & $\begin{array}{l}0.0 \\
0.3032 \\
2.074 \\
3.127 \\
3.722 \\
3.850 \\
\end{array}$ & $\begin{array}{l}0.0 \\
0.012217 \\
0.074969 \\
0.072804 \\
0.084777 \\
0.087320 \\
\end{array}$ & 6 \\
\hline $\begin{array}{l}83 \\
\text { Bi }\end{array}$ & $\begin{array}{l}.015175 \\
.015175 \\
.090526 \\
.090526 \\
.090526 \\
.090526 \\
\end{array}$ & $\begin{array}{l}0.244 \\
1.0 \\
2.832 \\
3.924 \\
4.535 \\
4.678 \\
\end{array}$ & $\begin{array}{l}0.0 \\
0.3084 \\
2.059 \\
3.103 \\
3.687 \\
3.824 \\
\end{array}$ & $\begin{array}{l}0.0 \\
0.012598 \\
0.077106 \\
0.074812 \\
0.087179 \\
0.089805 \\
\end{array}$ & 6 \\
\hline
\end{tabular}

TABLE I (CONE'd)

\begin{tabular}{|c|c|c|c|c|c|}
\hline$\underline{z}$ & & $\Leftrightarrow$ & $\underline{X}$ & $I$ & $f$ \\
\hline $\begin{array}{l}84 \\
80 \\
0\end{array}$ & $\begin{array}{l}.015665 \\
.015665 \\
.093105 \\
.093105 \\
.093105 \\
.093105 \\
\end{array}$ & $\begin{array}{l}0.246 \\
1.0 \\
2.801 \\
3.883 \\
4.486 \\
4.631 \\
\end{array}$ & $\begin{array}{l}0.0 \\
0.3122 \\
2.035 \\
3.071 \\
3.648 \\
3.787 \\
\end{array}$ & $\begin{array}{l}0.0 \\
0.012987 \\
0.079291 \\
0.076861 \\
0.089627 \\
0.092350 \\
\end{array}$ & \\
\hline $\begin{array}{l}85 \\
\text { AE }\end{array}$ & $\begin{array}{l}.016163 \\
.025253 \\
.095730 \\
.095730 \\
.095730 \\
.095730 \\
\end{array}$ & $\begin{array}{l}0.248 \\
1.0 \\
2.778 \\
3.852 \\
4.448 \\
4.592 \\
\end{array}$ & $\begin{array}{l}0.0 \\
0.3266 \\
2.020 \\
3.049 \\
3.620 \\
3.750 \\
\end{array}$ & $\begin{array}{l}0.0 \\
0.013763 \\
0.082516 \\
0.078945 \\
0.092120 \\
0.094937 \\
\end{array}$ & 6 \\
\hline $\begin{array}{l}86 \\
\text { in } \\
\end{array}$ & $\begin{array}{l}.016670 \\
.016670 \\
.058404 \\
.098606 \\
.098404 \\
.098404 \\
\end{array}$ & $\begin{array}{l}0.269 \\
1.0 \\
2.744 \\
3.799 \\
4.385 \\
4.530 \\
\end{array}$ & $\begin{array}{l}0.0 \\
0.3207 \\
1.992 \\
3.003 \\
3.564 \\
3.703 \\
\end{array}$ & $\begin{array}{l}0.0 \\
0.013784 \\
0.083785 \\
0.081067 \\
0.094661 \\
0.097573 \\
\end{array}$ & 6 \\
\hline $\begin{array}{l}87 \\
78 \\
7\end{array}$ & $\begin{array}{l}.027191 \\
.027191 \\
.101137 \\
.201137 \\
.101137 \\
.102137 \\
\end{array}$ & $\begin{array}{l}0.250 \\
1.0 \\
2.719 \\
3.765 \\
4.344 \\
4.487 \\
\end{array}$ & $\begin{array}{l}0.0 \\
0.3232 \\
1.972 \\
2.975 \\
3.530 \\
3.667 \\
\end{array}$ & $\begin{array}{l}0.0 \\
0.014296 \\
0.086207 \\
0.083233 \\
0.097256 \\
0.100264 \\
\end{array}$ & 6 \\
\hline 8 & $\begin{array}{l}.017722 \\
.017722 \\
.103922 \\
.103922 \\
.103922 \\
.103922 \\
\end{array}$ & $\begin{array}{l}0.250 \\
1.0 \\
2.699 \\
3.735 \\
4.312 \\
4.454 \\
\end{array}$ & $\begin{array}{l}0.0 \\
0.3378 \\
2.959 \\
2.956 \\
3.508 \\
1.664 \\
\end{array}$ & $\begin{array}{l}0.0 \\
0.014616 \\
0.088476 \\
0.085436 \\
0.099902 \\
0.102999 \\
\end{array}$ & 6 \\
\hline $\begin{array}{l}89 \\
\text { Ae } \\
\text { ? }\end{array}$ & $\begin{array}{l}.028266 \\
.018265 \\
.206759 \\
.106759 \\
.106759 \\
.106759 \\
\end{array}$ & $\begin{array}{l}0.251 \\
1.0 \\
2.676 \\
3.705 \\
4.276 \\
4.420 \\
\end{array}$ & $\begin{array}{l}0.0 \\
0.3288 \\
1.938 \\
2.926 \\
3.474 \\
3.612 \\
\end{array}$ & $\begin{array}{l}0.0 \\
0.015049 \\
0.090889 \\
0.087676 \\
0.102599 \\
0.105783 \\
\end{array}$ & 6 \\
\hline $\begin{array}{l}90 \\
\text { Th }\end{array}$ & $\begin{array}{l}.010920 \\
.018820 \\
.209651 \\
.109651 \\
.209651 \\
.209651 \\
\end{array}$ & $\begin{array}{l}0.252 \\
1.0 \\
2.651 \\
3.676 \\
4.241 \\
1.383 \\
\end{array}$ & $\begin{array}{l}0.0 \\
0.3322 \\
1.917 \\
2.901 \\
3.443 \\
3.579 \\
\end{array}$ & $\begin{array}{l}0.0 \\
0.015489 \\
0.093351 \\
0.089958 \\
0.105348 \\
0.108626 \\
\end{array}$ & 6 \\
\hline $\begin{array}{l}91 \\
70 \\
*\end{array}$ & $\begin{array}{l}.019384 \\
.019384 \\
.112601 \\
.112601 \\
.112601 \\
.122601 \\
\end{array}$ & $\begin{array}{l}0.256 \\
1.0 \\
2.625 \\
3.639 \\
4.196 \\
4.336 \\
\end{array}$ & $\begin{array}{l}0.0 \\
0.3335 \\
1.694 \\
2.867 \\
3.602 \\
3.536\end{array}$ & $\begin{array}{l}0.0 \\
0.015939 \\
0.095868 \\
0.092287 \\
0.108155 \\
0.111522 \\
\end{array}$ & 6 \\
\hline 92 & $\begin{array}{r}.019959 \\
.019959 \\
.115606 \\
.115606 \\
.125606 \\
.115606 \\
\end{array}$ & $\begin{array}{l}0.256 \\
2.0 \\
2.602 \\
3.606 \\
4.150 \\
4.296 \\
\end{array}$ & $\begin{array}{l}0.0 \\
0.3350 \\
1.875 \\
2.036 \\
3.370 \\
3.503 \\
\end{array}$ & $\begin{array}{l}0.0 \\
0.016398 \\
0.098436 \\
0.094658 \\
0.111015 \\
0.114485 \\
\end{array}$ & 6 \\
\hline
\end{tabular}


TABLE I (Cone'd)

\begin{tabular}{|c|c|c|c|c|c|}
\hline$z$ & $\varepsilon$ & 8 & $\underline{y}$ & $E$ & $\underline{\varepsilon}$ \\
\hline $\begin{array}{l}93 \\
98\end{array}$ & $\begin{array}{l}.020547 \\
.020547 \\
.118670 \\
.218670 \\
.118670 \\
.118670\end{array}$ & $\begin{array}{l}0.257 \\
2.0 \\
2.575 \\
3.562 \\
4.111 \\
4.247\end{array}$ & $\begin{array}{l}0.0 \\
0.3358 \\
1.851 \\
2.800 \\
3.328 \\
3.459\end{array}$ & $\begin{array}{l}0.0 \\
0.016967 \\
0.101057 \\
0.097070 \\
0.113931 \\
0.117503\end{array}$ & 6 \\
\hline $\begin{array}{l}94 \\
P u\end{array}$ & $\begin{array}{r}.021147 \\
.022247 \\
.121797 \\
.121797 \\
.122797 \\
.221797\end{array}$ & $\begin{array}{l}0.256 \\
2.0 \\
2.559 \\
3.509 \\
4.046 \\
4.280\end{array}$ & $\begin{array}{l}0.0 \\
0.3370 \\
1.319 \\
2.732 \\
3.269 \\
3.398\end{array}$ & $\begin{array}{l}0.0 \\
0.017348 \\
0.103734 \\
0.099527 \\
0.116905 \\
0.120583\end{array}$ & 6 \\
\hline
\end{tabular}

Eleaent osteted in prevtous vereion. 